\title{
Hydroxyapatite/polymer biocomposite scaffold as drug carrier
}

\author{
Pranabesh Sasmal ${ }^{1}$, Howa Begam ${ }^{*}$, Sarmita Sinha ${ }^{2}$, Bablu Sikder $^{2}$ and Abhijit Chanda ${ }^{2}$ \\ ${ }^{1}$ School of Bioscience and Engineering, Jadavpur University, Kolkata, India \\ ${ }^{2}$ Department of Mechanical Engineering, Jadavpur University, Kolkata, India \\ *Email: howabegam@gmail.com
}

Type I collagen is the main component of bone tissue. It has excellent biocompatibility and low immunogenicity as biomaterial, and is widely used as tissue scaffold for bone regeneration. Type I collagen, in conjunction with calcium-phosphatebased ceramics, is used as composite bone grafts. Biocomposites of hydroxyapatite (HAp) in combination with different polymers such as poly vinyl alcohol (PVA), chitosan, sodium alginate and collagen have the potential of serving in various tissue engineering applications, such as in bone repair and reconstruction. These composites are considered one of the most promising biomaterials as sustain drug carrier and bone implant due to its excellent biocompatibility. Biocomposite of HAp and collagen with polymeric binder can provide improved durability and mechanical properties.

The aim of this study was extraction of type-1 collagen from fish skin and in-situ synthesis of HAp/Collagen composite. Acid soluble collagen was isolated from sea fish skin at $4^{\circ} \mathrm{C}$. HAp/ collagen composite powder was synthesized using wet chemical method at room temperature. The extracted collagen and the HAp/collagen composite were characterized for XRD, FTIR, particle size distribution and SEM. From the FTIR analysis it was observed that the extracted collagen is type 1 collagen which consists of Amide groups. Peaks for collagen as well as HAp are present in HAp/collagen composite powder. SEM microstructure of collagen shows flake like structure. HAp powders synthesized are phase pure and the HAp/collagen composite powders are also phase pure.

HAp/ collagen composite film was prepared using other binders such as chitosan, PVA, sodium alginate. Different concentrations of polymeric binder were used during film preparation. Antiinflammatory drug diclofenac sodium was incorporated into HAp/polymer composite film prepared using PVA chitosan and Na-alginate. The composite film was also studied for FTIR, SEM (Figure 1). The drug release from the composite film in phosphate buffer solution was studied for a span of 30 days using Franz diffusion cell at $\mathrm{pH}$ 7.4 .
Data obtained from in vitro release studies were fitted to various kinetic equations to find out the mechanism of drug release (Figure 2). The kinetic models used were zero order model, first order model, Higuchi model and Korsemeyer- Peppas model.

The drug entrapment efficiency of formulation was found to be highest for $3 \%$ chitosan and lowest for $5 \%$ PVA. The in-vitro drug release was studied in phosphate buffer solution by varying the concentration of polymer. It was observed that the drug entrapment efficiency of formulation was found to be highest for $3 \%$ chitosan and lowest for $5 \%$ PVA and 3\% PVA is more efficient than chitosan according to Korsmeyer-Peppas model.

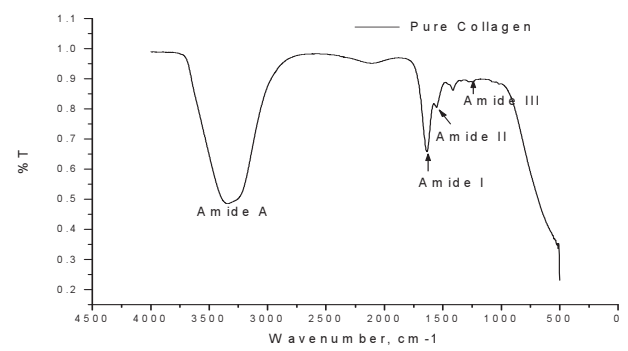

Figure 1: FTIR spectra of Collagen-1 extracted from sea fish skin

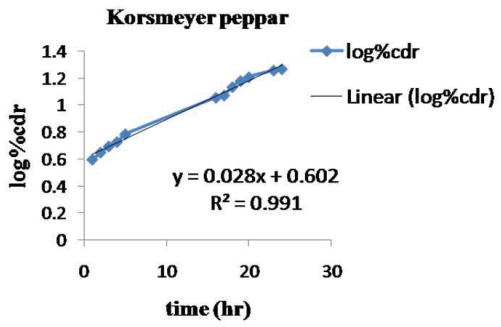

Figure 2: In vitro drug release from $3 \%$ PVA/HAp/Collagen/Drug film

\section{References}

1. M. H. Santos, L. G. D. Heneine, H. S. Mansur, Materials Science and Engineering C 28 (2008) 563-571 2. N. Degirmenbasi, D. M. Kalyon, E. Birinci, Colloids and Surfaces B: Biointerfaces 48 (2006) 42-49

3. Z. Li, B. Wang, C. Chi, Q. Zhang, Y. Gong, J. Tang, H. Luo, G. Ding, Food Hydrocolloids 31 (2013) 103113 